\title{
Indicateurs et déterminants sociaux de l'insécurité dans la ville de Lubumbashi
}

\author{
Delphin KAIMBI MPYANA*
}

\section{INTRODCTION}

Le débat sur l'insécurité et la sécurité des personnes et des biens est pareil à celui de l'ordre et du désordre en société. Cette dernière ne peut connaitre le progrès que lorsqu'elle a la capacité de transformer toutes situations en opportunité pour l'ordre à établir. C'est donc en embrassant la sécurité c'est-à-dire l'ordre qu'un Etat peut maintenir ses membres en état des droits.

Un Etat des droits n'est que l'antithèse de l'insécurité. Cette dernière constitue l'expression de l'anarchie à telle enseigne qu'une proposition du genre : A mesure que l'insécurité augmente, l'Etat des droits s'affaiblit ou encore l'Etat de droit est inversement proportionnel à la sécurité des personnes et des biens. Plus il y a la sécurité plus la société s'affirme comme un Etat des droits et vice-versa.

Quoi de plus normal que les détenteurs du pouvoir puissent s'investir à prendre des mesures visant à maintenir ou à protéger l'ordre ou la sécurité dans les villes comme celle de Lubumbashi où la concentration de la population et des activités diverses reste patente.

La ville de Lubumbashi en République Démocratique du Congo, comme modèle de la présente étude connait une recrudescence de l'insécurité ces dernières années. La population obligée à un certain moment de se prendre en charge dans une sorte de mouvement d'autodéfense populaire ou aux travers les pratiques qui, elles aussi, deviennent dans la suite insécurisantes; car non-formelles : la population fait de patrouilles nocturnes comme les forces de l'ordre; dort à l'extérieur surveillant les entrées et sorties de chaque rue, avenue ou quartier... chacun contrôle chacun.

Quels sont alors les indicateurs et déterminants sociaux de l'insécurité à Lubumbashi et qui affectent la matérialisation d'un Etat de Droit? Cette question constitue l'épicentre de notre réflexion. Pour la cohérence de notre raisonnement, il sera question dans un premier moment de faire un récapitulatif sur la genèse de la ville de Lubumbashi; en second lieu, de dégager les indicateurs de l'insécurité dans la ville de Lubumbashi; et ensuite d'identifier des déterminants sociaux à la base de l'insécurité dans la ville de Lubumbashi.

Enfin il sera question de réfléchir sur comment gérer l'insécurité dans la ville de Lubumbashi afin de contribuer à l'établissement d'un Etat des droits en République Démocratique du Congo. 


\section{A. De la genèse de la ville de Lubumbashi}

"Née de l'industrialisation coloniale, la ville de Lubumbashi est devenue aujourd'hui un lieu d'attraction d'une population en âge d'activités $»{ }^{1}$

Dès sa création, la vocation première de la ville fût celle de l'exploitation minière. Ainsi, ne pouvait y habiter que celui qui avait un emploi relatif à l'exploitation minière. Ville masculine à cause de sa main d'œuvre composée d'hommes recrutés partout à travers le Congo et en Afrique.

Selon Gabriel Kalaba Mutabusha ${ }^{2}$, la naissance de Lubumbashi est à relier à la question de trois compagnies : le Comité Spécial du Katanga (CSK) en 1900; 1’Union Minière du Haut-Katanga en 1906 et la Compagnie des Chemins de Fer du Bas-Congo au Katanga (BCK). Ces trois compagnies créées à Bruxelles, ont joué un rôle fondamental dans la création et le développement de Lubumbashi. Jusqu'aux années 1990 et sous de nouvelles dénominations ${ }^{3}$, elles ont continué à exercer une grande influence sur le développement de cette ville tant sur le plan économique, démographique que de l'habitat.

Ainsi, Lubumbashi est une ville des salariés dès son origine. Le salariat fut une condition d'accès à une résidence permanente. Le développement de la ville a été fortement lié à celui du travail salarié qui a atteint son apogée vers la fin des années 1950. En d'autres termes, sa sécurité était étroitement liée au travail salarié, intégrateur dans cette société industrielle et salariale.

\section{B. Indicateurs de l'insécurité dans la ville de Lubumbashi}

« L'insécurité, considérée comme un état de ce qui n'est pas sûr, qui n'offre pas de sécurité $»^{4}$, de garantie se traduit à travers des indicateurs bien précis.

Dans la ville de Lubumbashi, ce sentiment de danger, d'incertitude constaté se manifeste dans la ville de Lubumbashi à travers les éléments indicatifs ci-après :

- Le chômage croissant;

- L'accès difficile au logement décent;

- Le déficit alimentaire;

- La délinquance;

- Une faible prise en charge sanitaire;

1 KAIMBI MPYANA, « La marginalisation sociale urbaine à Lubumbashi : une contestation de l'ordre économique dominant », in Revue Gabonaise de Sociologie, n 8 , Paris, L'Harmattan, 2015.

2 Kalaba Mutabusha Gabriel, « La conception de l'autorité chez les Lambas d'aujourd'hui », in Revue du CEPAC, 1985, pp.12 - 13.

3 L'U.M.H.K., (L'Union Minière du Haut-Katanga) est devenue la Générale des Carrières et des Mines (GCM); la BCK est devenue Société Nationale des Chemins de fer du Zaïre (SNCZ).

4 Larousse Encyclopédique en couleurs, 11 France Loisirs, Paris, 1978. 
- Faible assurance de la retraite;

- Vols-viols.

\section{Le chômage croissant}

La ville de Lubumbashi a réussi à atteindre le plein emploi dans les années avant l'indépendance (1960) et quelques années après l'indépendance, car «pendant les premières décennies de l'existence de la ville, le pouvoir colonial mit en place une politique de recrutement massif et de stabilisation des travailleurs $\gg .{ }^{5}$

Dans une étude exhaustive sur 14000 ménages en 2006 dans les 7 communes que compte la ville de Lubumbashi, il a été constaté sur les non-salariés les résultats suivants :

"Commune annexe 78,9\%; commune Kamalondo 66\%; commune Kampemba 59,6\%; commune Katuba 73,3\%; commune Kenya 75,2\%; commune Lubumbashi 53,8\%; commune Ruashi 53,3\%. Dans l'ensemble, Lubumbashi qui en 1973 comptait 60,7\% des salariés et en 1958 pas moins de 94\% des salariés; aujourd'hui la ville a paradoxalement plus de non-salariés : $65,3 \% \ldots »^{6}$

Aujourd'hui, cette situation n'a pas connue de changement majeur. Par contre elle s'est empirée. Il y a de plus en plus une population active surtout dans les centres urbains et donc à Lubumbashi sans emploi et qui est obligée de trouver des solutions au problème de survie dans une ville d'origine salariale.

\section{L'absence de logement}

Le problème de logement est un indicateur réel qui accroit le sentiment de danger.

De plus en plus l'on remarque les longs des grandes artères de la ville de Lubumbashi, un nombre croissant d'enfants à l'âge de scolarité sans domicile. Certains passent nuit dans des aubettes ou des habitations abandonnées. Ceux qui sont d'un âge avancé trouvent refuge dans les marchés publics.

Le logement est un véritable casse-tête pour beaucoup de familles qui consacrent une part importante de leur revenu. Après l'éducation, l'alimentation vient le logement comme poste budgétaire le plus couteux pour l'habitant de Lubumbashi.

\section{Le déficit alimentaire}

En octobre 2012, une enquête de la FAO sur la consommation des ménages à Lubumbashi est arrivée aux résultats suivants : « la consommation moyenne des habitants de la ville est de 1335 calories et de 40,5 gr de protéines par personne et par jour. Par rapport aux stan-

5 Lire Bruneau J.C., Lubumbashi, capitale du Cuivre. Ville et citadins au Zaïre, thèse de Doctorat en Géographie, Université de Bordeaux III, 1990, pp-385-395.

6 C. Nkuku et M. Remon, Stratégies de survie à Lubumbashi, paris, L'Harmattan, 2006, p.61. 
dards établis par les nutritionnistes pour une vie active et saine 2300 calories et $70 \mathrm{~g}$ de protéines par jour et par personne.

Seules les communes de Lubumbashi et de Ruashi sur les six communes que compte la ville ont une consommation dépassant les 1500 calories par jour et par personne.

D'autres études montrent aussi que $80 \%$ des familles mangent une (1) fois par jour en fonction de la rente journalière des membres de la famille (enfants et parents).

Selon le même rapport, moins de $20 \%$ des familles interrogées ont une provision d'au moins un aliment de base, $80 \%$ se débrouillent au jour le jour en tout.

\section{Une faible prise en charge sanitaire ${ }^{7}$}

Les déficits quantitatifs et qualitatifs de l'offre des services publics, en particulier dans les domaines de la santé publique et de l'éducation (...), face à une demande sociale de plus en plus croissante numériquement et quantitativement, plus exigeante et plus complexe, ont ouvert un large champ d'opportunités de commercialisation de ces services.

Cette situation a entre autres deux conséquences sociales. La première est la tendance croissante à la privation d'un service public qui relève du domaine des obligations de l'Etat en tant que droit des citoyens, dans un pays où l'assurance maladie n'est pas encore droit pour les citoyens; en second lieu la marchandisation croissante de la santé amène à la concentration des capacités de soins de santé dans les centres urbains et autres concentrations humaines où existe le « marché » des services de santé, c'est-à-dire la demande solvable.

Aux termes des décisions d'Abuja, les pays se sont engagés à allouer au moins $15 \%$ de leur budget public aux dépenses de la santé.

La République Démocratique du Congo a des peines à respecter cet engagement.

Cette moyenne a atteint 11,4\% en 2012 avant de dégringoler jusqu'à 9\% en 2015.

\section{La délinquance}

La délinquance, fait observable dans la ville de Lubumbashi frappe plus les jeunes à l'âge de scolarité. Ils sont de plus en plus nombreux à passer leur temps à déambuler et à mendier et à commettre des petits forfaits çà et là.

En effet, comme il y a une association forte entre la délinquance et la scolarité, il a été remarqué au travers le rapport national sur le développement humain 2016 que le taux net de scolarisation dans la province du Katanga était relativement faible par rapport aux autres provinces.

Il est présentement « le secteur de l'éducation devenant surtout un secteur pour l'investissement privé, et donc un domaine pour des affaires aussi juteuses que d'autres $» .{ }^{8} \mathrm{C}$ 'est

7 PNUD, RNDH 2016 : Croissance inclusive, développement durable et défi de la décentralisation en République Démocratique du Congo, Août 2017, p.16.

8 PNUD, op.cit., p.20. 
l'ère de marchandisation de l'éducation en République Démocratique du Congo. Devant cette situation, l'école devient producteur des exclus du système éducatif, des délinquants.

\section{Vols-viols}

Dans un seul quartier de la ville de Lubumbashi (quartier Kalebuka) les statistiques suivantes ont été prélevées auprès de la police.

Infraction selon les statistiques de la police

\begin{tabular}{|c|c|c|c|c|c|c|c|}
\hline \multirow{2}{*}{$\mathbf{N}^{\circ}$} & Année & \multirow{2}{*}{\multicolumn{2}{|c|}{2015}} & \multirow{2}{*}{\multicolumn{2}{|c|}{2016}} & \multirow{2}{*}{\multicolumn{2}{|c|}{2017}} \\
\hline & \multirow[t]{2}{*}{ Nature des infractions } & & & & & & \\
\hline & & Effectif & $\%$ & Effectif & $\%$ & Effectif & $\%$ \\
\hline 1 & Vols simples & 295 & 0,25 & 326 & 0,26 & 434 & 0,36 \\
\hline 2 & Escroquerie & 49 & 0,04 & 50 & 0,04 & 193 & 0,16 \\
\hline 3 & Viols & 8 & 0,01 & 8 & 0,01 & 133 & 0,11 \\
\hline 4 & Vols par effraction (v.e) & 228 & 0,20 & 245 & 0,20 & 112 & 0,09 \\
\hline 5 & Attaque à mains armées & 78 & 0,07 & 74 & 0,06 & 75 & 0,06 \\
\hline 6 & Violences et voies de faits & 181 & 0,16 & 197 & 0,16 & 54 & 0,04 \\
\hline 7 & Abus de confiance $(\mathrm{ABC})$ & 136 & 0,12 & 150 & 0,12 & 50 & 0,04 \\
\hline 8 & Coups et blessures volontaires & 59 & 0,05 & 63 & 0,05 & 5 & 0,00 \\
\hline 9 & Meurtres & 1 & 0,00 & 4 & 0,00 & 1 & 0,00 \\
\hline 10 & $\begin{array}{l}\text { Autres (drogue, faux billets, recel, } \\
\text { ivresse publique) }\end{array}$ & 125 & 0,11 & 133 & 0,11 & 154 & 0,13 \\
\hline & TOTAL & 1160 & 1,00 & 1250 & 1,00 & 1211 & 1,00 \\
\hline
\end{tabular}

Source : rapport de la police, Quartier Kalebuka, 2017

Hier comme aujourd'hui, ce tableau montre que les viols et les vols sont des infractions récurrentes dans la ville de Lubumbashi hier comme aujourd'hui.

\section{Faible assurance de la retraite}

Le régime de sécurité en République Démocratique du Congo ne prend en compte que les travailleurs, il est professionnaliste.

Alors que la majeure partie de la population (80\%) vit sous le régime de l'économie de la débrouille; il est quasi impossible qu'il soit pris en charge par la caisse de sécurité à l'âge de dépendance. 
Raison pour laquelle, les meilleurs investissements pour le futur sont les biens et non les enfants qui passées pour une charge au temps présent. Acquérir un bien de valeur que l'on peut revendre en cas de nécessité (maladie,...) même à vil prix, est plus rassurant.

Ainsi contrairement à la tradition en vogue au début de la création de la Gécamines et donc de la ville de Lubumbashi, qui incitait les travailleurs à avoir beaucoup d'enfants (futur main d'œuvre locale) pour un équivalent en biens et avantages sociaux; de plus en plus une nouvelle tradition s'est mise en place en place : il faut disposer de plusieurs biens matériels pour assurer son avenir.

"Ainsi, une chute temporaire du revenu peut être amortie, voire compensée par la possession d'un logement ou la vente d'actifs détenus sous forme de biens. Ce capital réduit la vulnérabilité des ménages aux chocs subis (perte d'emploi, inflation, etc.) "9

\section{Déterminants sociaux de l'insécurité}

Plusieurs facteurs concourent à la recrudescence de l'insécurité dans la ville de Lubumbashi. Nous avons regroupé les différents facteurs en deux catégories :

- Facteurs institutionnels;

- Individualisation des réponses aux problèmes collectifs.

\section{Facteurs institutionnels}

Les institutions sont les meilleurs instruments que l'homme a forgé pour vivre en harmonie en société.

Ces institutions qui, généralement jouent un rôle intégrateur par l'imposition des normes; des manières d'agir et de penser, sont devenues soient obsolètes soient inadaptées au contexte de la nouvelle ville surpeuplée et dont le marché de travail est très réduit.

La famille face aux aspects manifestes de pauvreté a cessé de jouer son premier rôle d'institution primaire de socialisation. Les liens familiaux sont soumis à l'épreuve de la satisfaction optimale des besoins.

L'école est devenue un lieu d'exclusion sociale pour les moins nantis. Il n'est pas aisé d'envoyer et de maintenir l'enfant à l'école durant toute la période de scolarité. Rien n'est gratuit à l'école et les parents doivent prendre en charge le fonctionnement de l'école et instruction des enfants.

L'église, une autre institution inadaptée par son caractère publicitaire « des faiseurs des miracles » et son coté marchand qui se rependent dans beaucoup d'églises actuelles dans la ville de Lubumbashi. Les exclus qui s'y refugient s'ils ne sont pas qualifiés de sorciers, ils sont considérés comme possédés par les esprits impurs; et doivent par ricochet être délivrés.

9 Makabu Ma NKENDA, et Alii, Rapport sur l'emploi, le chômage et les conditions d'activité en République Démocratique du Congo : Principaux résultats de la phase 1 et de l'enquête 1-2-3, 20042005, IRD, DT/2007-14. 
L'Etat, son affaiblissement au travers ses instruments de contrôle et de contraintes (police, armée, appareils judiciaires) ne lui permet pas de jouer au sein de la population son rôle de protecteur des personnes et de leurs biens.

La population vit dans une sorte d'anomie. Les institutions ne peuvent plus jouer leurs fonctions de base. Ce qui contribue à l'accroissement de l'insécurité dans la ville de Lubumbashi.

\section{Individualisation des réponses aux problèmes collectifs}

Les problèmes de pauvreté; de réalisation des besoins fondamentaux ou secondaires sont des problèmes collectifs dans une société en perpétuel désarticulation entre une prévention budgétaire et sa réalisation.

Face donc à cette situation; la population de Lubumbashi a tendance à apporter des réponses individuelles. Par conséquent, la contrainte sociale, est sur plantée par les exigences individuelles de service. L'individu vit non en tenant compte des autres; mais pour son propre compte comme dans la jungle où la loi reste : la lutte pour la vie.

Une telle manière d'agir accroit l'insécurité de chacun vis-à-vis de l'autre. L'individualisme et la faible intégration sociale qui les caractérisent sont à la base d'une nouvelle forme de suicide, que E. Durkheim appelle suicide anomique (un suicide par affaiblissement des normes sociales).

\section{Comment gérer l'insécurité à Lubumbashi pour un Etat de Droit}

Il y a une nécessité de trouver de solutions durables à l'insécurité grandissante dans la ville. Pour y parvenir nous proposons que les détenteurs du pouvoir se sensibilisent et sensibilisent la population à l'éthique de responsabilité, car l'anarchie est partout quand la responsabilité n'est nulle part.

L'Ethique de responsabilité repose sur le souci de trouver des solutions, des réponses aux questions de la société non en comptant sur les autres mais sur soi-même.

Il est question ici d'intérioriser la question principale : « quelle est ma part dans cette situation-problème? », " Quelle est ma contribution dans ce projet du mieux-être de la population?

C'est en ramenant chacun dans sa sphère de responsabilité à comprendre et à accepter son rôle que chaque animateur des institutions ci-dessus identifiés; chaque acteur sociétal devra jouer son rôle pour le bien-être de tous.

Ceci veut dire aussi qu'au-delà de l'Etat de Droit, il devient impérieux que chacun puisse penser à un Etat de Devoir comme pour paraphraser René Dekkers dans son discours de la rentrée académique à l'Université de Lubumbashi : « Je suis persuadé que l'humanité 
entière serait moins agitée si on ne lui parlait pas tous les jours de ses droits; et beaucoup plus de ses devoirs $»{ }^{10}$

En somme, l'insécurité dans la ville de Lubumbashi fait suite à l'inadéquation entre la vocation première de la ville (ville salariale) et afflux incontrôlé de la population dans ce centre urbain. Ce dernier incapable de donner des réponses aux préoccupations de ses habitants fait de ceux-ci, des exclus, des marginaux et donc des délinquants prêts à tout. La solution se trouve dans l'éthique de responsabilité.

\section{BIBLIOGRAPHIE}

Bruneau J.C., Lubumbashi, capitale du Cuivre. Ville et citadins au Zaïre, thèse de Doctorat en Géographie, Université de Bordeaux III, 1990.

C. Nkuku et M. Remon, Stratégies de survie à Lubumbashi, paris, L'Harmattan, 2006.

Dekkers, R., Pour une déclaration universelle des devoirs de l'homme, in Discours Rectoraux, Lubumbashi, 1969.

FAO, Coordination des opérations agricoles d'urgence, Enquête sur la consommation des ménages, octobre 2012.

Kaimbi Mpyana, La marginalisation sociale urbaine à Lubumbashi : une contestation de l'ordre économique dominant in Revue Gabonaise de Sociologie, nº, Paris, L'Harmattan, 2015.

Kalaba Mutabusha Gabriel, «La conception de l'autorité chez les Lambas d'aujourd'hui », in Revue du CEPAC, 1985.

L'U.M.H.K., (L’Union Minière du Haut-Katanga) est devenue la Générale des Carrières et des Mines (GCM); la BCK est devenue Société Nationale des Chemins de fer du Zaïre (SNCZ).

Larousse Encyclopédique en couleurs, 11 France Loisirs, Paris, 1978.

Makabu Ma NKENDA, et Alii, Rapport sur l'emploi, le chômage et les conditions d'activité en République Démocratique du Congo : Principaux résultats de la phase 1 et de l'enquête 1-2-3, 20042005, IRD, DT/2007-14

PNUD, Rapport National de Développement Humain 2016, Août 2017 croissance inclusive, développement durable et défi de la décentralisation en République Démocratique du Congo.

PNUD, RNDH 2016: Croissance inclusive, développement durable et défi de la décentralisation en République Démocratique du Congo, Août 2017.

10 Dekkers, R., Pour une déclaration universelle des devoirs de l'homme, in Discours Rectoraux, Lubumbashi, 1969. 